\title{
RISKY DRIVING BY RECENTLY LICENSED TEENS: SELF-REPORTS AND SIMULATED PERFORMANCE
}

\author{
Teresa M. Senserrick, ${ }^{1}$ Timothy Brown, ${ }^{2}$ Dawn Marshall, ${ }^{2}$ \\ D. Alex Quistberg, ${ }^{1}$ Ben Dow, ${ }^{2}$ Flaura K. Winston ${ }^{1,3}$ \\ ${ }^{1}$ Center for Injury research and Prevention \\ The Children's Hospital of Philadelphia \\ Philadelphia, PA, USA \\ E-mail: senserrick@email.chop.edu \\ ${ }^{2}$ Cognitive Systems Team \\ National Advanced Driving Simulator \\ Center for Computer Aided Design \\ University of Iowa \\ Iowa City, Iowa, USA \\ E-mail: tbrown@nads-sc.uiowa.edu \\ ${ }^{3}$ Division of General Pediatrics, Leonard Davis Institute \\ University of Pennsylvania \\ Philadelphia, PA, USA
}

\begin{abstract}
Summary: U.S. teens are overrepresented in motor vehicle crashes, with the majority due to driver error; however, causal pathways remain to be elucidated. This research aimed to identify driving performance factors that might underlie newly-licensed male teens' risk. Surveys were conducted with 21 16-year-olds at the time of intermediate licensure. During the second month of licensure they completed drives in a high-fidelity simulator. Simulator scenarios allowed assessment of responses to yellow traffic lights changing to red and to a visual search task, for which previous data on older age groups of drivers were available. All teens had an A or B grade point average, previously found to be associated with lower crash and citation risk. Nonetheless, 71\% reported risky driving in terms of prior unlicensed, unsupervised driving. In the simulator, $46 \%$ went through an intersection as the light turned red, compared to $33 \%$ of adults. In the visual search task, teens had shorter mean perception-reaction times and identified more targets than adults and older drivers, but similar to young drivers. Therefore, even teens with good grades, perceived to be less risky, were willing to take driving risks. Their driving performance suggests there may be subtle differences in the way recently-licensed teens drive that might predispose them to crashes. Further research of this nature can increase understanding of such differences and inform the development of more targeted intervention.
\end{abstract}

\section{INTRODUCTION}

Like many developed countries worldwide, U.S. teenagers are overrepresented in motor vehicle crashes (IIHS, 2006). In 2005, 3,467 15-20 year-old drivers were killed. The majority ( 80\%) of the fatality crashes involved driver error, moreso than speeding or alcohol (Williams et al, 2005). While much is known about factors that contribute to teen driver risk (Shope, 2006), the precise causal pathways to crashes are unknown. 
One major contributing factor is inexperience. Risk is extreme, irrespective of age, during the first and second month (Maycock et al., 1991; Mayhew et al., 2003), declines rapidly through to six months and less rapidly but steadily through to 12 months, but remains higher than for experienced drivers for several years, with a similar pattern occurring by increasing miles travelled (McCartt et al., 2003; Williams, 2003). Risky driving behavior is also a contributor (Gregersen \& Bjurulf, 1996; Maycock, 2002; Neyens \& Boyle, 2007), with young males in particular found to drive more quickly, more aggressively, have a greater propensity to violate rules and react more quickly in traffic; thereby leaving smaller margins of error (Maycock, 2002). In addition, young driver crashes commonly occur at intersections (Berg et al., 2004), and teens with a $\mathrm{C}$ or $\mathrm{D}$ grade point average have been shown to have a higher risk of citation and crash risk risk than teens with an A or B grade point average (McCarrt et al., 2003). Therefore, all novices are at risk, with the first months of independent driving, males, intersections and grade point averages, being key area of focus for research.

This study aimed to identify driving performance factors underlying inflated crash risk of newlylicensed male teens by comparing their performance in simulator drives, which included intersection scenarios, to that found previously for young, adult and older driver age groups.

\section{METHODS}

\section{Equipment and Materials}

Survey. A survey was conducted in order to describe the sample and explore the heterogeneity of participants. It was programmed and published on the NADS website with SurveyPro Netcollect 3.1 (Apian Software, Inc., 2005). Access was restricted by sign-on username and passwords.

In addition to demographic questions, items included: "When did you get the following driving permit or license? Instructional Permit [learner's permit], Minor School License [Iowa license that allows unsupervised driving to/from school only] (Month/Year, Do not have)"; and "Have you ever driven in the following vehicles and situations? Thinking of the last 30 days, on how many days did you drive the following? Car, Other passenger vehicle, Motorcycle, Moped or ATV, Farm vehicle, Other (Supervised/ Unsupervised)."

A sensation-seeking measure was included as a proxy for likely driving risk-taking since, as new drivers, direct measures of driving behaviors were likely inappropriate. High sensation seeking, a personality trait characterized by a desire for novelty and intensity of sensory stimulation (Zuckerman, 1990), has been found to correlate significantly with driving while intoxicated, driving over $80 \mathrm{mph}$, driving $20 \mathrm{mph}$ over the speed limit, racing other drivers, and passing in no-passing zones (Arnett, 1996). The previously validated, reliable Brief Sensation Seeking Scale (Hoyle et al., 2002) was included, which comprises 8 items: (1) I would like to explore strange places, (2) I would like to take off on a trip with no pre-planned routes or timetables, (3) I get restless when I spend too much time at home, (4) I prefer friends who are exciting and unpredictable, (5) I like to do frightening things, (6) I would like to try bungee jumping, (7) I like wild parties, and (8) I like new and exciting experiences, even if I have to break the rules ( $1=$ Strongly disagree, $2=$ Disagree, $3=$ Neither disagree or agree, $4=$ Agree, $5=$ Strongly Agree) . 
Simulator drives. The NADS is an advanced high-fidelity ground simulator with a 13-degreesof-freedom motion system previously validated for exploring teen driver speed management on rural roads (Senserrick et al., under review). The study protocol was leveraged from a previous (unpublished) National Highway Traffic Safety Administration project that examined driver performance of young (18-25), middle, (30-45), and older (50-60) aged driver groups. Participants encounter a variety of situations while navigating the environment, including traffic lights that turn red as the driver approaches and a visual search task.

The yellow-light task involved a traffic light changing from green to yellow to red as the participant approached the intersection. Two different timings were evaluated: one in which the light changes to yellow when the driver is 3 seconds from the stop line, and the second when the driver is 3.75 seconds from the stop line. For this analysis, the variable of interest was whether the driver went through the light or not.

In the visual search task, the driver had to search for a target pedestrian along the road (see Figure 1 for example). The objective was to identify pedestrians that had a gold "I" on a black shirt when it appeared in the environment (far left in Figure 1). The targets appeared at either 250 or 350 feet in front of the driver (longitudinal Figure 1. Example of a scene with a target pedestrian distance) and either 25 or 50 feet to the left or the right of the center of the right lane of the road (lateral distance). The variables of interest were correct responses to targets and perception-reaction time to the targets.

\section{Participants and Procedure}

The current study was part of a larger project studying teens and parents. Recruitment drives were conducted via flyer distribution and presentations at schools in 12 local school districts, newspaper advertisements and an information booth at a local mall, reaching approximately 1,000 teens. Potential participants were directed to the NADS web page, which provided requirements, downloads of consent forms or requests for printed versions and contact details for further information. Teens were required to be aged 15-16 years, possess a learner permit and intend to possess an intermediate license by August 31, 2006. The aim of these inclusion criteria was to control (at least in part) for effects of age and intermediate licensed driving experience.

As male teens, on average, have different risk-taking and driving experience than female teens, only male teens in the larger project were invited to participate in the simulator (and companion on-the-road study). To further ensure that the male participants were novices, subject recruitment was limited to those who were not engaged in full-time employment requiring driving. Participants also had to pass a battery of health and medication-related screening criteria required by the NADS facility. An additional Informed Consent document describing the simulator study was required (which also included an on-the-road drive). 
Of the 73 teens who qualified and enrolled in the project, $45(61.6 \%)$ were male. Once the NADS protocol was ready to run, all 33 male teens who responded to the survey at that time were invited to participate in the simulator study. Of those invited, $21(63.6 \%)$ participated. The mean age was 16.1 years (range 16.0 to 16.7$)$. Only $1(4.8 \%)$ participant identified as Hispanic. The majority (18 or $85.7 \%$ ) was White/Caucasian, with 1 (4.8\%) Black/African American, and 2 $(9.5 \%)$ were of mixed race. All participants had an A or B grade point average $(\mathrm{M}=3.39$, $\mathrm{SD}=0.44)$.

The survey was completed at the time of licensure and the simulator drive, totaling approximately 1 hour, on a single visit to the NADS during the second month of licensure. Participants were requested to drive normally and safely. An experimenter rode in the rear seat to give route instructions. Immediately following simulator drives, participants answered questions regarding simulator disorientation symptoms to ensure they were not experiencing any such disorientation.

Monetary incentives were offered for participation, as well as the opportunity to drive a sports car simulator at the NADS facility at the end of the broader project (several months later). The complete project protocol was approved on full review by the Institutional Review Board at the University of Iowa, and administrative review at The Children's Hospital of Philadelphia.

\section{RESULTS}

Four (19.0\%) of the 21 participants had held a Minor School License for an average of 7.04 months (range 1.57 to 11.04 ) prior to intermediate licensure. Nonetheless, all remaining participants reported prior unsupervised driving, with $5(41.7 \%)$ reporting driving unsupervised during the previous 30 days. This suggested all participants had some baseline, although variable, unsupervised driving experience prior to intermediate licensure.

Participant sensation-seeking scores are displayed in Figure 2. There was a spread of scores from lower to higher sensation seeking, suggesting heterogeneity among participants.

Responses to the yellow-light event were examined using a chi-squared analysis. When looking at overall differences associated with how drivers responded to the changing light, a significant difference $\left[\chi^{2}(3)=8.64, p<0.05\right]$ was found amongst the four driver age groups. As can be seen

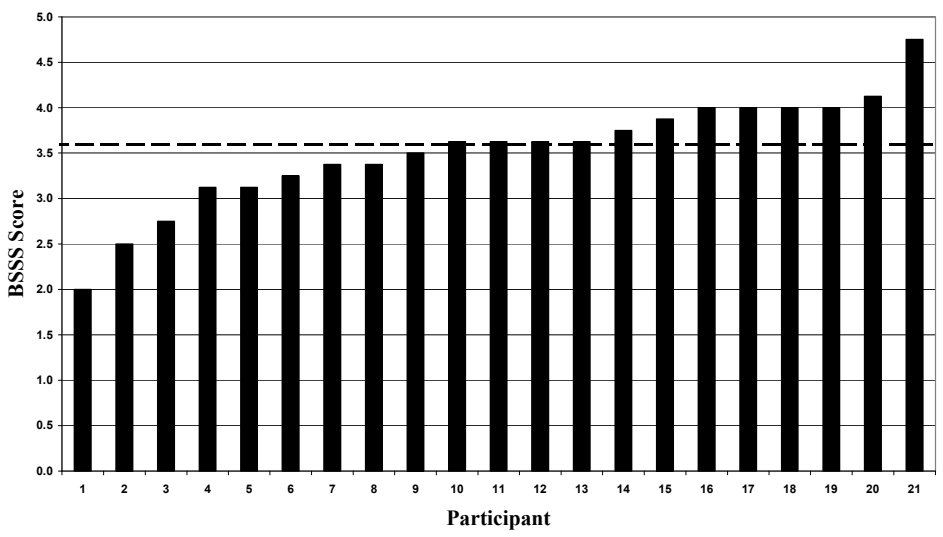

Figure 2. Participant sensation seeking scores with median split

in Figure 3 for All Lights, the novice and older drivers went through more lights than did their young and middle-age driver counterparts. When examining the two timings separately, there was no statistical difference for the 3-second timing $\left[\chi^{2}(3)=2.63, p>0.05\right]$; however, there was a statistical difference for the 3.75-second timing $\left[\chi^{2}(3)=9.90, p<0.05\right]$. As can be seen in Figure 3, for the 3.75-second timing, it appears 
that although novice drivers run about $10 \%$ more lights than their young and middle-aged counterparts, they run about $10 \%$ fewer lights than the older drivers.

For reaction time to targets in the visual search task, we first examined only targets that were correctly identified. When driver age group was analyzed together with the lateral and longitudinal distances to the targets, there was no statistical difference in reaction time across the driver age groups $[\mathrm{F}(3,40)=0.82, \mathrm{p}=0.49]$. Second, we examined cases where the driver failed to respond, treating the reaction times as the total time that the target had been visible to the driver. For example for the targets that appeared 250 feet in front of the driver, the value of 3.78 seconds was used (the amount of time elapsed at $45 \mathrm{mph}$ in $250 \mathrm{feet}$ ). So instead of treating the value as missing, it could be replaced with this conservative estimate of how long it would have taken the driver to respond. When driver age group was analyzed together with the lateral and longitudinal distances to the targets using this approach, a significant difference was found for driver age group $[\mathrm{F}(3,40)=8.22, \mathrm{p}<0.01]$.

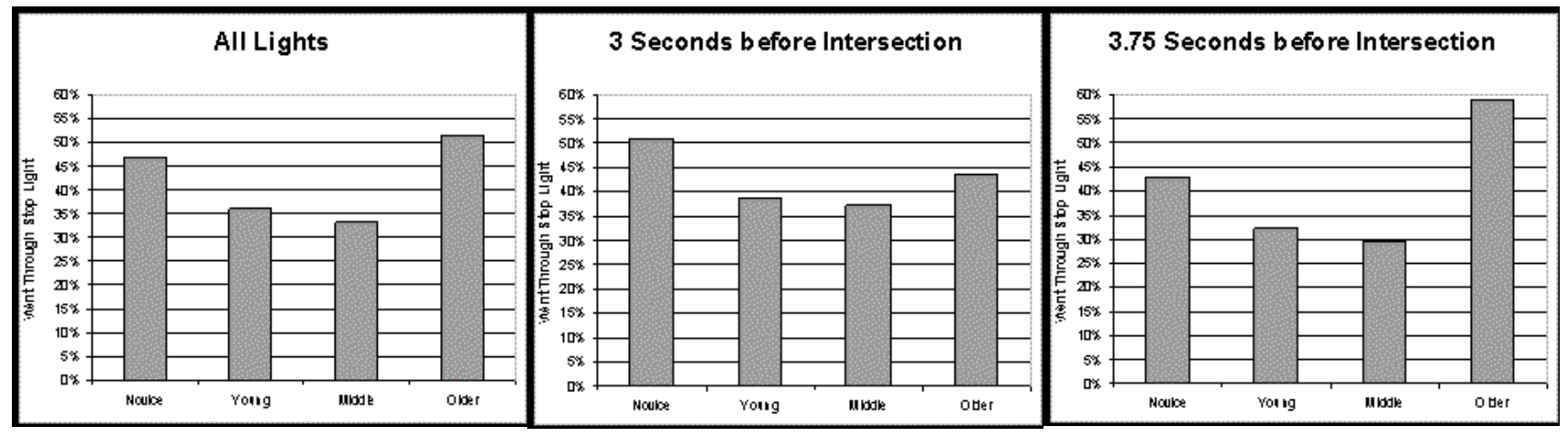

Figure 3. Driver response to light changing to yellow on approach

Figure 4 illustrates the differences between groups. A Tukey post-hoc test revealed that the novice and young driver age groups responded faster than the middle- or older aged drivers, and that the older drivers responded slower than the middle-aged drivers.

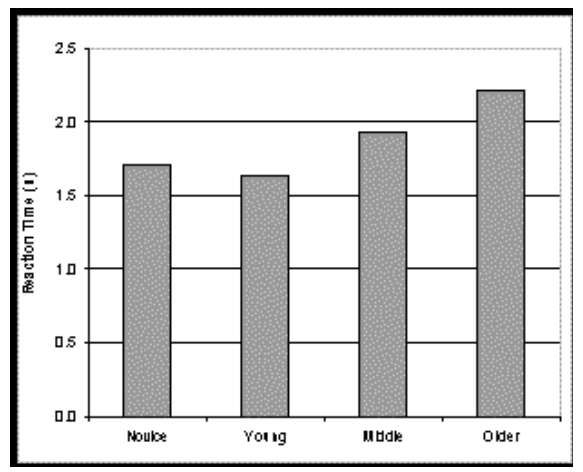

Figure 4. Visual search target reaction time

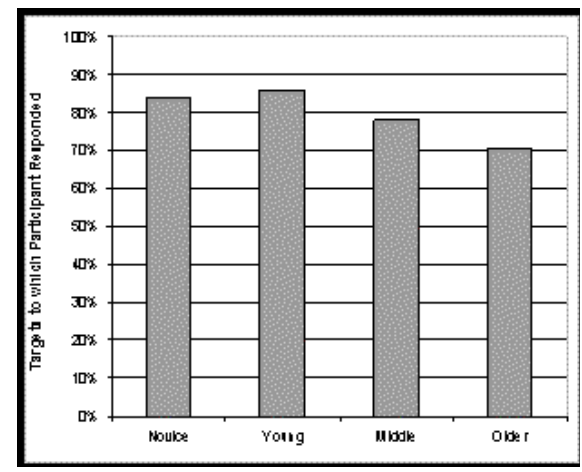

Figure 5. Visual search target detection

For the response to the targets, shown in Figure 5, there was a statistically significant difference in response between the four driver age groups $\left[\chi^{2}(3)=287.60, p<0.001\right]$. With young and novice drivers responding correctly most frequently followed by middle-aged drivers and older drivers. 


\section{CONCLUSIONS}

Despite good grades, which have previously been associated with reduced citations and crashes (McCarrt et al., 2003), the male teens in our study were willing to take driving risks, as found in self reports of prior unlicensed, unsupervised driving and in riskier behavior in the driving simulator compared to previous findings with more experienced young, adult and older drivers.

The interaction with traffic control devices through the drive is of particular safety concern, and novice drivers were more prone to run through lights for which there was sufficient time to stop than their counterparts in the young and middle-aged driver groups. Nonetheless, as shown in a previous simulator study (De Winter et al., 2007), careful analysis is needed before concluding whether this performance manoeuver represented intentional violations versus driver error. Overall, novice drivers ran about as many lights as did their older driver counterparts. Although the results are similar in terms of outcome for the older and novice drivers, it would seem that the manifestation of this behavior is different. It seems likely based on the fact that the youngdriver group, which should have a similar reaction time to the novice group, was able to stop more frequently, that the novice group is either not attending to the lights as much as the youngdriver group or is not able to judge as well when they have sufficient time to stop; whereas the older driver group may be affected by the onset of slowed reaction times associated with aging.

Interpreting the visual search task results is a little more complicated. Overall, novice drivers responded as fast as or faster than the other driver age groups and correctly identified as many or more targets than did their counterparts. Initial analyses of the first five participants only showed a much different response to the visual search task than did the final analysis of all 21 participants' data. Initially it appeared that although novice drivers would respond faster, they would do so at the expense of missing more targets. That trend did not hold true through full data collection. It is possible that two or more different groups of novice drivers that behave differently are included in our data set [e.g., the small subgroup $(n=4)$ of drivers with extensive driving experience on a Minor School License]; however, our small participant numbers do not provide enough power for subgroup analyses. Future analyses will focus on better understanding how the novices behave relative to other data we have collected.

The main limitation of this study is the small participant number. The spread in sensationseeking scores suggests there was heterogeneity among participants, which is preferable for the purpose of generalizability, however, may also have contributed to the inconsistent findings in relation to the visual search task. These findings are not generalizable to other rural male teen populations or other teens in general. Data was utlized from participants that were not all run in the same study context or during the same time period, however, it is not anticipated that this affected responses.

The visual search task, although requiring many of the same skills drivers use when navigating through the streets, is not a task that would normally be undertaken in the real world. Due to the constant scanning and non-standard target location, it may exacerbate driver age differences and penalize more experienced drivers who have developed an efficient scanning pattern for normal driving activities; however, this task is prone to identify issues associated with changes in the environment that are not associated with "normal" day-to-day driving. 
These findings suggest there are subtle differences in the way recently-licensed teens drive that might predispose them to crash risk. Further research of this nature can increase understanding of such differences and inform the development of more targeted intervention to help reduce the unacceptably high incidence of fatal crashes among teens.

\section{ACKNOWLEDGMENTS}

The authors would like to acknowledge the funding and support of the National Science Foundation, with special thanks to the Center for Child Injury Prevention Studies Industrial Advisory Board members. We also acknowledge Ginger Watson, PhD, original Principal Investigator at NADS, who contributed significantly to the study design and early stages, and the succeeding Principal Investigator, Omar Ahmad, for his contribution during the later stages.

\section{REFERENCES}

Berg, H.-Y., Gregersen, N.P., \& Laflamme, L. (2004). Typical patterns in road-traffic accidents during driver training: An explorative Swedish national study. Accident Analysis \& Prevention, 36, 603-608.

De Winter, J.C.F., Wieringa, P.A., Kuipers, J., Mulder, J.A., \& Mulder, M. (2007). Violations and errors during simulation-based driver training. Ergonomics, 50(1), 138-158.

Gregersen, N.P. \& Bjurulf, P. (1996). Young novice drivers: Towards a model of their accident involvement. Accident Analysis \& Prevention, 28(2), 229-246.

Hoyle, R.H., Stephenson, M.T., Palmgreen, P., Lorch, E.P., \& Donohew, R.L. (2002). Reliability and validity of a brief measure of sensation seeking. Personality and Individual Differences, 32, 401-414.

IIHS. (2006). Fatality Facts 2005: Teenagers. Insurance Institute for Highway Safety. Retrieved November 21, 2006, from http://www.iihs.org/research/fatality_facts/teenagers.html

Maycock, G. (2002). Novice driver accidents and the driving test. TRL Report 527, Crowthorne UK: Transport Research Laboratory.

Maycock, G., Lockwood, C.R., \& Lester, J.F. (1991). The accident liability of car drivers. TRL Research Report 315. UK: Transport Research Laboratory.

Mayhew, D.R., Simpson, H.M., \& Pak, A. (2003). Changes in collision rates among novice drivers during the first months of driving. Accident Analysis \& Prevention, 35, 683-691.

McCartt, A.T., Shabanova, V.I., \& Leaf, W.A. (2003). Driving experience crashes and traffic citations of teenage beginning drivers. Accident Analysis \& Prevention, 35, 311-320.

Neyens, D.M. \& Boyle, L.N. (2007). The effect of distractions on the crash types of teenage drivers. Accident Analysis \& Prevention, 39, 206-212.

Senserrick, T.M., Brown, T., Quistberg, D.A., Marshall, D., Ahmad, O., \& Winston, F.K. (2007). Validation of simulated assessment of teen driver speed management on rural roads. Paper submitted to $51^{\text {st }}$ Annual Scientific Conference of the Association for the Advancement of Automotive Medicine (AAAM), Melbourne, Australia, October 14-18. 
Shope, J.T. (2006). Influences on youthful driving behavior and their potential for guiding interventions to reduce crashes. Injury Prevention, 12 (Suppl 1), i9-i14.

Williams, A.F. (2003). Teenage drivers: patterns of risk. Journal of Safety Research, 34, 5-15.

Williams, A.F., Ferguson, S.A., \& Wells, J.K. (2005). Sixteen-year-old drivers in fatal crashes, United States, 2003. Traffic Injury Prevention, 6(3), 202-206. 